\title{
Long-Term Study of Children With ROME III Functional Gastrointestinal Disorders Managed Symptomatically in a Biopsychosocial Model
}

\author{
Shailender Madani ${ }^{\mathrm{a}, \mathrm{d}}$, Suchi Parikh ${ }^{\mathrm{b}}$, Rohit S. Madanic, \\ Amornluck Krasaelap ${ }^{\mathrm{a}}$
}

\begin{abstract}
Background: Our study evaluated progression of and identified potential factors contributing to outcomes of ROME III defined-functional gastrointestinal disorders (FGIDs) in children treated symptomatically in a biopsychosocial model of care with a long-term follow-up.

Methods: We performed a retrospective review of pediatric patients who were diagnosed with ROME III defined-FGIDs including functional abdominal pain, functional dyspepsia, irritable bowel syndrome and abdominal migraine. Patients were managed symptomatically in a biopsychosocial model of care from the time of initial diagnosis. Demographics, management, progression and response to treatment assessed as complete, partial, and no improvement were reviewed.
\end{abstract}

Results: Two hundred fifty-eight patients were included with mean age of 10.6 years, female $55.4 \%$, mean number of encounters 3.3 visits, and mean follow-up was 18.7 months (range 2 - 59, SD 15.8). Diagnoses were functional abdominal pain $45 \%$, irritable bowel syndrome $20.9 \%$, multiple $13.2 \%$, functional dyspepsia $12.8 \%$, and abdominal migraine $8.1 \%$. Investigations were performed in most patients: laboratory studies in $93.4 \%$ (non-contributory abnormal $23.6 \%$ ), imaging studies in $45.3 \%$ (non-contributory abnormal $5 \%$ ) and endoscopies in $43.0 \%$ (non-contributory abnormal $1.2 \%$ ). Treatment included medication in $93.7 \%$, and surgery in $1.9 \%$ (normal pathology). There were new functional gastrointestinal diagnosis in $11.6 \%$, evolution of FGIDs, from one to another in $12.0 \%$, and recurrence found in $35.7 \%$ of patients. There were $60.1 \%$ patients in the complete improvement group (CIG) and $39.1 \%$ in the partial/no

Manuscript accepted for publication February 07, 2017

aThe Carman Ann Adam Department of Pediatrics, Wayne State University School of Medicine, Children's Hospital of Michigan, 3901 Beaubien Blvd, Detroit, MI 48201, USA

bCook Children's Physician Network, 2755 Miller Ave, Forth Worth, TX 76105, USA

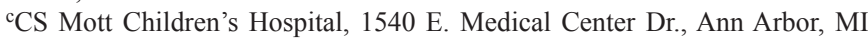
48109, USA

${ }^{\mathrm{d} C}$ Corresponding Author: Shailender Madani, The Carman Ann Adam Department of Pediatrics, Wayne State University School of Medicine, Children's Hospital of Michigan, 3901 Beaubien Blvd, Detroit, MI 48201, USA.

Email: smadanimd@gmail.com

doi: https://doi.org/10.14740/gr798w improvement group (PIG/NIG). No statistical difference was found between CIG and PIG/NIG regarding demographics or evaluation. PIG/NIG had more encounters (mean 3.63 vs. 3.11; $\mathrm{P}=0.03$ ), had non-contributory lab abnormalities $(34.4 \%$ vs. $20.0 \%$; $\mathrm{P}=0.01)$, needed more endoscopies $(52.4 \%$ vs. $36.8 \% ; \mathrm{P}=0.02)$, required more treatment changes (mean 1.41 vs. $0.81 ; \mathrm{P}<0.01$ ) and developed new functional gastrointestinal diagnoses $(19.4 \%$ vs. $6.5 \%$; $>0.01)$ with long-term follow-up.

Conclusions: Patients with ROME III defined-FGIDs who experience partial or no improvement with treatment develop new FGID diagnosis, need more number of follow-up visits, require more number of endoscopies, need more treatment changes, and have more non-contributory laboratory abnormalities, compared to those who experience complete improvement. Symptomatic treatment offered in a biopsychosocial model of care is possibly beneficial in managing children with FGIDs.

Keywords: ROME criteria; Functional gastrointestinal disorders; Biopsychosocial model; Long-term follow-up

\section{Introduction}

Functional gastrointestinal disorders (FGIDs) present a diagnostic, and treatment challenge in the subspecialty, and primary care setting. They account for more than $50 \%$ of the consultations in pediatric gastroenterology practice and $2-4 \%$ of all general pediatric office visits [1]. Children with FGIDs have lower health-related quality of life than those with an organic gastrointestinal disease [2]. Recently, more than half of all new patients presenting to the pediatric gastroenterology clinic met the ROME III criteria for one or more FGIDs [3].

Despite the widespread use of ROME criteria over a decade, it has not substantially changed the evaluation or treatment practices for children with chronic abdominal pain among primary care physicians and pediatric gastroenterologists $[4,5]$. Additionally, functional disorders run a prolonged course for more than 5 years as reported in a large US household survey [6]. A prospective study found up to $41 \%$ of children had clinically significant abdominal pain after a mean duration of a 9-year follow-up into adolescence and young adulthood [7]. The treating physician faces the conundrum of a missed di- 
agnosis that explains the patient's symptoms. This has to be balanced with making a positive diagnosis of an FGID, reducing pain, improving patient/parent satisfaction with care and decreasing health care utilization. The American Academy of Pediatrics (AAP) subcommittee on chronic abdominal pain in conjunction with the North American Society of Pediatric Gastroenterology Hepatology and Nutrition (NASPGHAN) recommended that children with chronic abdominal pain, who lack alarm signs or symptoms to suggest an organic disease, be evaluated within the primary care setting in a biopsychosocial model, and be offered symptomatic treatment to reduce the frequency or severity of symptoms [8]. The aim of our study was to evaluate progression and identify potential factors contributing to outcomes of ROME III defined-FGIDs in children treated symptomatically in a biopsychosocial model of care with a long-term follow-up.

\section{Materials and Methods}

A retrospective chart review included all children (4 - 21 years) who presented with abdominal pain associated conditions and were diagnosed with ROME III criteria based FGID, namely functional abdominal pain (FAP), functional dyspepsia (FD), irritable bowel syndrome (IBS), and abdominal migraine (AM). At the time of diagnosis and follow-up, symptomatic treatment was offered to these children in a biopsychosocial model of care. This is a standard of care model practiced by the principal investigator based on the principles of empathy, validation, education, reassessment, and negotiation [9], adapted from documented guidelines in the literature [10].

The symptoms were explained to the parent and patient in a simple, age appropriate language that although the pain is real, there is most likely no serious underlying disease [8]. For all of these patients, the treatment goal was the prompt return to school, and participation in daily function, by addressing any potential impediments. Assisting the patient to cope was via judicious use of medications for symptomatic relief, and/ or offering psychological intervention as clinically warranted. Individualized management plans were made, and the collaboratively chosen therapy was initiated.

The study was approved by the institutional review board of Children's Hospital of Michigan. Charts were reviewed for evidence-based [11, 12] and anecdotally used symptomatic treatments that are known to be beneficial: cyproheptadine, dicyclomine, hyoscyamine, acid reducers, amitriptyline/ nortriptyline, citalopram, peppermint oil, Iberogast, aloe vera, coenzyme CoQ 10, probiotics, cognitive behavioral therapy, hypnotherapy, guided imagery singly, and/or in combination. Patients who did not receive any medication were also included in the study because reassurance about the condition has been reported to be therapeutic [13]. At a subsequent followup, response was assessed by a subjective improvement in pain as reported by the patient, and/or parent. A child's self report is generally considered to be the "gold standard" for pain assessment, when used in conjunction with observer's report of pain is considered a valuable indication of treatment outcome in both clinical, and research contexts [14-16]. More re- cently, patient-reported outcome measures of gastrointestinal symptoms are recommended to determine treatment effect on FGIDs like IBS and FAP [17]. Response to one or more symptomatic treatments was categorized and recorded as: complete improvement group (CIG: improvement allowing for discontinuation of medication, or parental willingness to come off the medication, with or without recurrence of symptoms requiring ongoing or intermittent use) or partial improvement group/no improvement group (PIG/NIG: patients requiring increased dose of medication or addition of another medication, and/or intervention). This was done at follow-up visit in gastrointestinal (GI) clinic, emergency room visit, a parent phone call to the GI office, or follow-up phone call by the gastroenterologist. All parents have a direct phone access to the treating gastroenterologist.

Each FGID case was reviewed for documentation of demographic data, FGID diagnosis (overlap diagnosis and developing new diagnosis), number of clinic encounters, lab testing, endoscopies, and any clinical changes. Results on performance of: lab studies (CBC, ESR, albumin, tissue transglutaminase antibodies, quantitative IgA level, amylase, lipase, ALT, AST, alkaline phosphatase, fecal occult blood, stool ova and parasite), imaging (abdominal X-ray, abdominal ultrasound, abdominal CT, hepatobiliary scan, abdominal MRI, upper gastrointestinal series with small bowel follow-through), endoscopic studies (upper endoscopy and colonoscopy) whether already performed prior to, or ordered after the consultation and surgeries as part of treatment of pain were recorded, along with response to treatment and development of new symptoms. Any documentation for development of a new diagnosis that explained the cause of pain resulting in improvement of symptoms was recorded during the longest follow-up period for every patient.

\section{Inclusion criteria}

All patients with chronic abdominal pain who met the ROME III criteria for FGIDs [18] with at least a 1-month post-consultation follow-up were included.

\section{Exclusion criteria}

We excluded patients with any alarm symptoms or physical findings at initial encounter suggestive of an organic etiology like unexplained fever, unexplained weight loss, hematemesis, rectal bleeding, protracted vomiting, chronic diarrhea, persistent right upper or right lower quadrant pain, and abnormal physical findings, such as localized fullness, hepatomegaly, splenomegaly, costovertebral angle tenderness or spinal tenderness, and perianal abnormalities. Patients who were subsequently diagnosed with organic diseases or patients with no follow-up data were also excluded.

\section{Statistical analysis}

Data were presented using descriptive statistics. Categorical 
Table 1. Demographic Information of All Patients With FGIDs

\begin{tabular}{|c|c|}
\hline $\mathrm{N}$ & 258 \\
\hline Mean age, years (range, SD) & $10.6(4-18,3.9)$ \\
\hline Female & $143(55.4 \%)$ \\
\hline \multicolumn{2}{|l|}{ Race } \\
\hline Caucasian & $180(69.8 \%)$ \\
\hline African American & $46(17.8 \%)$ \\
\hline Middle Eastern & $18(7.0 \%)$ \\
\hline Unknown & $8(3.1 \%)$ \\
\hline Hispanic & $6(2.3 \%)$ \\
\hline \multicolumn{2}{|l|}{ Initial FGIDs } \\
\hline FAP & $116(45.0 \%)$ \\
\hline IBS & $54(20.9 \%)$ \\
\hline Multiple & $34(13.2 \%)$ \\
\hline FD & $33(12.8 \%)$ \\
\hline AM & $21(8.1 \%)$ \\
\hline \multicolumn{2}{|l|}{ Number of initial FGID diagnoses } \\
\hline 1 & $224(86.8 \%)$ \\
\hline$>1$ & $34(13.2 \%)$ \\
\hline Mean duration of symptoms in months (range, SD) & $22.1(1-135,25.8)$ \\
\hline Family history of FGID & $40(15.5 \%)$ \\
\hline First degree (parent or sibling) & $27(10.5 \%)$ \\
\hline Second degree & $13(5.0 \%)$ \\
\hline Family history of IBS & $37(14.3 \%)$ \\
\hline Family history of FGIDs other than IBS & $3(1.2 \%)$ \\
\hline Psychiatric disorder & $81(31.4 \%)$ \\
\hline Anxiety/anxiety symptoms & $58(22.5 \%)$ \\
\hline Multiple disorders & $14(5.4 \%)$ \\
\hline Depression & $8(3.1 \%)$ \\
\hline Bipolar & $1(0.4 \%)$ \\
\hline
\end{tabular}

FGIDs: functional gastrointestinal disorders; FAP: functional abdominal pain; IBS: irritable bowel syndrome; FD: functional dyspepsia.

data were presented as proportions and percentages. Continuous data were presented using means, range and standard deviations. Demographic data, investigations, results, treatment and outcome data of the CIG and PIG/NIG were compared. Differences in proportions of categorical variables were compared using a Fisher's exact Chi-square test. Differences in mean values of continuous variables were examined using a parametric independent sample t-test. All statistical procedures were performed using SPSS version 22.0 (IBM SPSS Statistics, Armonk, NY). Statistical significance was considered at a P-value $\leq 0.05$, two-tailed.

\section{Results}

Three hundred eight children were diagnosed with FGIDs based on ROME III criteria at their initial clinical encounter in the GI clinic. Thirty-nine patients were excluded from the analysis because of inadequate follow-up data. Eleven patients were also excluded as an organic disease developed during the follow-up period. The study included a total of 258 children with FGIDs. Demographic data including age, gender, race, diagnosis, duration of symptoms, follow-up period, family history and psychiatric disorder are provided in Table 1. Diagnostic tests including laboratory testing, imaging study, endoscopy and results are summarized in Table 2 . Treatment data including type of medication, supplements used, psychological treatment, and surgery are summarized in Table 3. Follow-up and outcome data of the study including number of encounters, response, recurrence and final FGID diagnoses are shown in Table 4. Evaluation, management and treatment outcome data of FGIDs in the CIG and PIG/NIG are compared in Table 5. 


\section{Discussion}

This is a descriptive/observational study reporting on a large cohort of children with FGIDs based on ROME III criteria, treated symptomatically (appropriate to their clinical condition) by a single physician in a biopsychosocial model of care, and followed on a long-term period.

We noted clustering of cases between $8-10$ and $15-17$ years of age. A systematic review in 2005 found evidence for a bimodal peak in the symptoms of recurrent abdominal pain being more prevalent between 4 and 6 years and preadolescence [19], compared to a 2015 study reporting a bimodal early peak at $5-7$ years and a late peak at $11-14$ years [20]. The significance of this secular trend in age at presentation is not well understood. We hypothesize that social changes and cultural beliefs over time since the original description by Apley [21] determine the response of the child to life experiences, and hence age at presentation. Additionally, the original premise of stressful life events may not be the only contributing factor.

In our study, the most common single diagnosis was FAP in $45 \%$, with more than one FGID diagnoses at initial consultation in $13.2 \%$. This finding compares with IBS being the most common in $37 \%$ and two diagnoses in $29 \%$ reported in a study that also applied ROME III criteria [22]. We hypothesize that this difference could be related to the understanding that FAP and IBS are possibly different expressions of one underlying disorder [23], or that the two are the most common FGID diagnoses, and a chance occurrence in our cohort. Moreover, the possibility of finding two FGIDs at diagnosis should be kept in mind while evaluating these patients.

Although almost all patients in our study underwent labo-
Table 2. Diagnostic Testing and Results of All Study Patients With FGIDs

\begin{tabular}{cl}
\hline $\mathrm{N}$ & 258 \\
Laboratory testing & $241(93.4 \%)$ \\
$\quad$ Normal & $180(69.8 \%)$ \\
Abnormal* & $61(23.6 \%)$ \\
Imaging study & $117(45.3 \%)$ \\
Normal & $104(40.3 \%)$ \\
Abnormal* & $13(5 \%)$ \\
Endoscopy & $111(43.0 \%)$ \\
EGD & $78(30.2 \%)$ \\
EGD and colonoscopy & $33(12.8 \%)$ \\
Normal & $108(41.9 \%)$ \\
Abnormal** & $3(1.2 \%)$ \\
\hline
\end{tabular}

FGIDs: functional gastrointestinal disorders. *Mildly abnormal and nonspecific laboratory value/imaging finding. ${ }^{* *}$ Parietal cell hyperplasia, basal cell hyperplasia and juvenile polyp (that do not explain the cause of pain).

ratory studies and no statistical difference of number of laboratory studies performed between the CIG and the PIG/NIG, there were significantly more abnormal laboratory values in the PIG/NIG. These abnormal laboratory results were mildly abnormal and non-specific, were considered insignificant/noncontributory, and thus disregarded, as they did not explain the cause of pain. We believe this is because repeated testing is bound to find non-specific, and potentially non-contributory

Table 3. Treatments Used for FGIDs

\begin{tabular}{cl}
\hline $\mathrm{N}$ & 258 \\
Received treatment & $245(95.0 \%)$ \\
Medications & $242(93.7 \%)$ \\
1 medication & $98(38.0 \%)$ \\
2 medications & $115(44.6 \%)$ \\
$>2$ medications & $29(11.2 \%)$ \\
Cyproheptadine & $124(48.1 \%)$ \\
Acid reducer & $118(45.7 \%)$ \\
Antispasmodic & $72(27.9 \%)$ \\
Laxative & $71(27.5 \%)$ \\
Loperamide & $8(3.1 \%)$ \\
CoQ10 & $4(1.6 \%)$ \\
Amitriptyline & $1(0.4 \%)$ \\
\hline Herbal supplements & $33(12.8 \%)$ \\
Psychological intervention & $31(12.1 \%)$ \\
Surgery & $5(1.9 \%)$ \\
Combination of treatments & $58(22.5 \%)$ \\
Number of treatment changes, mean (range, SD) & $1.05(0-6,1.2)$ \\
\hline
\end{tabular}

FGIDs: functional gastrointestinal disorders. 
Table 4. Follow-Up and Outcome Data of All Study Patients With FGIDs

\begin{tabular}{ll}
\hline $\mathrm{N}$ & 258 \\
Total number of encounters, mean (range, SD) & $3.32(1-12,1.84)$ \\
\hline Data available in months, mean (range, SD) & $18.7(2-59,15.8)$ \\
$\quad 1$ - 30 months & $201(77.9 \%)$ \\
$\quad>30-42$ months & $24(9.3 \%)$ \\
\hline Complete improvement & $33(12.8 \%)$ \\
\hline Partial improvement & $155(60.1 \%)$ \\
No improvement & $77(29.8 \%)$ \\
Recurrence & $26(10.1 \%)$ \\
New FGID diagnosis & $92(35.7 \%)$ \\
Total number of final FGID diagnoses & $30(11.6 \%)$ \\
$\quad 1$ diagnosis & \\
$\quad 2$ diagnoses & $197(76.4 \%)$ \\
\hline$\quad 3$ diagnoses & $55(21.3 \%)$ \\
$\quad 4$ diagnoses & $4(1.6 \%)$ \\
Evolution of FGID* & $2(0.8 \%)$ \\
Duration to final FGID diagnosis in months, mean (range, SD) & $31(12.0 \%)$ \\
\hline
\end{tabular}

*Most common evolution was IBS to FAP in seven, followed by FAP to IBS in six patients. FGIDs: functional gastrointestinal disorders; FAP: functional abdominal pain; IBS: irritable bowel syndrome; FD: functional dyspepsia; AM: abdominal migraine.

findings. Almost half of study patients underwent imaging studies and there was no difference in abnormal imaging result between the $\mathrm{CIG}$ and $\mathrm{PIG} / \mathrm{NIG}$, suggesting routine testing may not be beneficial.

Endoscopies were performed significantly more in the PIG/NIG as compared to the CIG but no difference in the result between these two groups was demonstrated. We hypothesize that endoscopies performed for reassurance about absence of disease in the PIG/NIG do not improve symptoms because of the level of anxiety related to persistence of symptoms among these patients.

Almost all patients received some form of symptomatic treatment along the lines of a biopsychosocial model of care, with complete improvement in symptoms noted in $60 \%$ children. Overall the most common medication used was cyproheptadine, followed by acid reducers. Cyproheptadine is reported to be effective in a prospective [24], retrospective [25] study and reported as holding promise in a systematic review [26] in FGIDs. These are treatments among the variety of available options used to manage symptoms of FGIDs [1, 23, 26]. In our study, there was no statistical difference noted in improvement in patients who received treatment including cyproheptadine, acid reducer, antispasmodic, laxative or herbal medication. The improvement noted in our study can arguably be attributed to the possibility of a placebo effect, and inherent bias of retrospective design. Nonetheless, in a double blind prospective multicenter study comparing children with FAP treated with amitriptyline compared to placebo, there was no difference between the groups [27]. However, a retrospective study without a placebo group demonstrated a 78.6\% response among patients treated with amitriptyline/imipramine for IBS, FAP, and FD [28]. The phenomenon of placebo response and its modifiers is not well understood.

A non-directive, non-judgmental, patient-centered history taking, provision of a clear explanation of the disorder along the lines of the parent/patient's beliefs, validation of the true nature of pain with empathy, inviting parent/patient to participate in collaboratively developing management plans, setting reasonable expectations, helped development of an improved doctor-patient relationship [29], and hence contributed to the symptom improvement. The mean number of encounters being significantly less in the CIG compared to PIG/NIG could be related to patient's satisfaction with treatment. We hypothesize that a biopsychosocial model of care could be one of the factors that may have played a role in the improvement of symptoms in our cohort. Given the fact that the patients in the PIG/ NIG also received a biopsychosocial model of care, and did not do well as their counterparts, we hypothesize there may be other factors contributing to outcomes and response to intervention in these FGID patients that are as yet not fully understood. This is also a possible explanation of the new finding in our study that patients in the PIG/NIG tend to develop new FGID diagnoses.

It is also interesting that five of our 258 patients ended up having surgery as a treatment for pain: two appendectomies and three cholecystectomies. All pathology reports were unremarkable and no improvement of symptoms was reported after surgeries. This suggests caution while surgery is being 
Table 5. Evaluation, Management and Treatment Outcome of FGIDs in Patients With and Without Complete Improvement

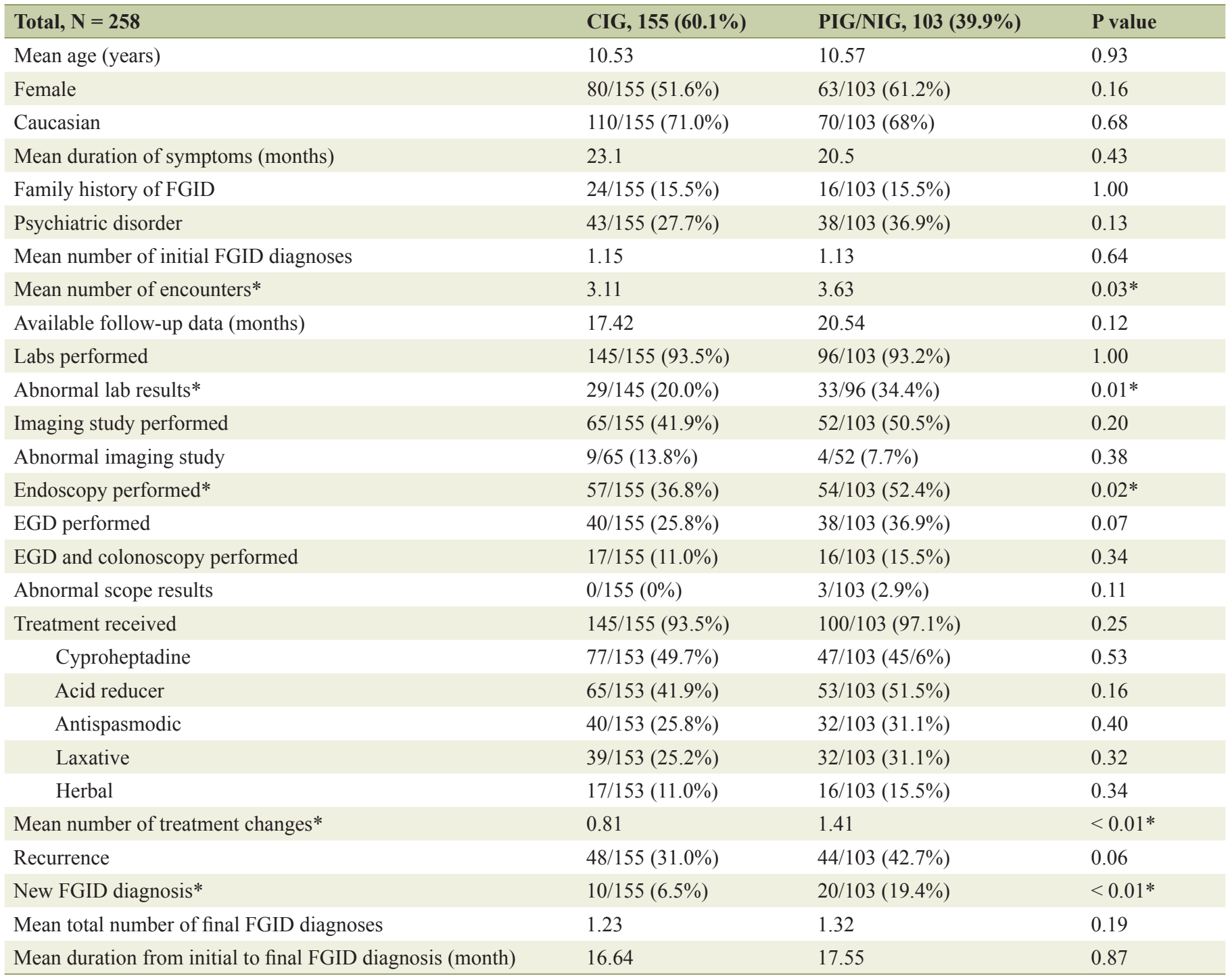

CIG: complete improvement group; PIG: partial improvement group; NIG: no improvement group; FGIDs: functional gastrointestinal disorders. *Entries and numbers represent statistically significant values.

considered as a therapeutic option.

At the end of our study, over three-fourths of patients had one FGID diagnosis, and almost one-fourth had more than one FGIDs. We found the most common diagnosis at the end of follow-up being FAP followed by IBS. In a prospective study of pediatric patients with FAP diagnosed per ROME III criteria found the most common FGID at the end of a 9-year followup to be IBS (17.6\%), followed by IBS with FD (10.5\%) [7]. We attribute this difference to paucity of outcome data in the literature from well-delineated trials.

Overlap of symptoms of FGID at diagnosis has been reported in children [30] with $33 \%$ of 142 patients in another study having overlapping FGID diagnoses. This compares with $13 \%$ of 258 patients in our cohort. In adults, this overlap was noted to range between $4 \%$ and $9 \%$ of subjects in a population-based study [31].
An adult study found subjects changing from one functional disorder to another over time [32] such as what we found evolving from one FGID to another in $10 \%$ of our cases suggesting they may be dynamic entities. A 12 -year US population-based cohort [33] reported change of diagnosis from IBS to FD. They extrapolated that there is an overlap in their underlying pathophysiology, which may have implications in terms of development of targeted therapies for common subgroups.

A systematic study found persistence of symptoms in $29.1 \%$ cases followed for a median of 5 years [34] that compares with a higher percentage (40\%) found in our study followed for a mean of 18.7 months. The reason for long-term persistence of FGIDs is not known. One of the associated risk factors reported is in relation to family history of FGIDs. A systematic review found moderate evidence for parental GI 
problems to be a risk factor for persistence of chronic abdominal pain in children [34]. A family history of GI disorders was reported in $15.4 \%$ cases in at least one parent in a recent study [20], whereas we had such a history among parents and siblings in $10.5 \%$ cases. Even though a correlation was not found in our study, this historical information is important to keep in mind in the evaluation of these patients.

In recent years, evidence of association between FGIDs and psychological disorders was reported in both primary care and referral center setting [35]. Even though we found a history of psychiatric disorders in $31.4 \%$ compared to $22.9 \%$ cases reported in a cross-sectional study [20], our study did not find a correlation between psychiatric disorder and improvement. This suggests a non-predominant role of psychiatric conditions in the response to treatment of FGIDs. However, in a biopsychosocial model of management, it is still pertinent for gastroenterologists to be aware of in developing individualized management plans in potentially improving outcomes.

There are recommendations in the literature regarding managing patients with FGIDs in a biopsychosocial model of care [9]. To date, there are no prospective or retrospective studies among pediatric patients addressing its application in the evaluation and management of children with FGIDs. Additionally, there have been no adult studies as well so far. To our knowledge, this study is the first in reporting on symptomatic treatment in conjunction with application of the biopsychosocial model of care in a retrospective pediatric cohort suffering from ROME III FGIDs. Based on the improvement observed amongst almost two-thirds of children (all of whom received some form of symptomatic treatment) in a large cohort over the range of FGIDs, we hypothesize this approach may have played a positive role in the outcome.

Strengths of our study are those of a descriptive/observational one [36]. There is no selection bias as only those children who met the criteria were included. This single physician application of the biopsychosocial model significantly diminishes practice variability, making it relatively consistent, possibly increasing credibility of the outcome and providing evidence of trends and tendencies in the course of FGIDs.

Limitations are those of a descriptive study: no comparison group, findings indicating norms not standards (as there is no comparison group), giving a limited picture of the cohort studied, and a limited generalizability by being a single physician experience. Also, documentation of pain improvement did not use a standard scale. The conclusions of the study need to be taken with caution.

\section{Conclusions}

Children with ROME III defined-FGIDs who experience partial or no improvement with treatment develop new FGID diagnosis, need more number of follow-up visits, require more number of endoscopies, need more treatment changes, and have more non-contributory laboratory abnormalities, compared to those who experience complete improvement. Symptomatic treatment offered in a biopsychosocial model of care is possibly beneficial in managing children with FGIDs. These findings need to be validated in rigorous analytical studies.

\section{Conflicts of Interest}

None.

\section{Source of Funding}

None.

\section{Author Note}

Statistical analysis: Dr Ronald Thomas (Statistitian) and Dr Amornluck Krasaelap.

\section{References}

1. Nurko S, Di Lorenzo C. Functional abdominal pain: time to get together and move forward. J Pediatr Gastroenterol Nutr. 2008;47(5):679-680.

2. Varni JW, Bendo CB, Nurko S, Shulman RJ, Self MM, Franciosi JP, Saps M, et al. Health-related quality of life in pediatric patients with functional and organic gastrointestinal diseases. J Pediatr. 2015;166(1):85-90.

3. Rouster AS, Karpinski AC, Silver D, Monagas J, Hyman PE. Functional Gastrointestinal Disorders Dominate Pediatric Gastroenterology Outpatient Practice. J Pediatr Gastroenterol Nutr. 2016;62(6):847-851.

4. Sood MR, Di Lorenzo C, Hyams J, Miranda A, Simpson P, Mousa H, Nurko S. Beliefs and attitudes of general pediatricians and pediatric gastroenterologists regarding functional gastrointestinal disorders: a survey study. Clin Pediatr (Phila). 2011;50(10):891-896.

5. Schurman JV, Hunter HL, Friesen CA. Conceptualization and treatment of chronic abdominal pain in pediatric gastroenterology practice. J Pediatr Gastroenterol Nutr. 2010;50(1):32-37.

6. Drossman DA, Li Z, Andruzzi E, Temple RD, Talley NJ, Thompson WG, Whitehead WE, et al. U.S. householder survey of functional gastrointestinal disorders. Prevalence, sociodemography, and health impact. Dig Dis Sci. 1993;38(9):1569-1580.

7. Horst S, Shelby G, Anderson J, Acra S, Polk DB, Saville BR, Garber J, et al. Predicting persistence of functional abdominal pain from childhood into young adulthood. Clin Gastroenterol Hepatol. 2014;12(12):2026-2032.

8. Di Lorenzo C, Colletti RB, Lehmann HP, Boyle JT, Gerson WT, Hyams JS, Squires RH, Jr., et al. Chronic abdominal pain in children: a clinical report of the American Academy of Pediatrics and the North American Society for Pediatric Gastroenterology, Hepatology and Nutrition. J Pediatr Gastroenterol Nutr. 2005;40(3):245-248.

9. Drossman DA. Functional abdominal pain syndrome. Clin Gastroenterol Hepatol. 2004;2(5):353-365.

10. Drossman DA. The functional gastrointestinal disorders and the Rome III process. Gastroenterology. 2006;130(5):1377-1390. 
11. Saps M, Di Lorenzo C. Pharmacotherapy for functional gastrointestinal disorders in children. J Pediatr Gastroenterol Nutr. 2009;48(Suppl 2):S101-103.

12. Clouse RE. Pharmacotherapy of altered brain-gut interactions in functional gastrointestinal disorders. J Pediatr Gastroenterol Nutr. 1997;25(Suppl 1):S18-19.

13. Hyams JS. Irritable bowel syndrome, functional dyspepsia, and functional abdominal pain syndrome. Adolesc Med Clin. 2004;15(1):1-15.

14. von Baeyer CL. Children's self-reports of pain intensity: scale selection, limitations and interpretation. Pain Res Manag. 2006;11(3):157-162.

15. Franck LS, Greenberg CS, Stevens B. Pain assessment in infants and children. Pediatr Clin North Am. 2000;47(3):487-512.

16. Chambers CT, Giesbrecht K, Craig KD, Bennett SM, Huntsman E. A comparison of faces scales for the measurement of pediatric pain: children's and parents' ratings. Pain. 1999;83(1):25-35.

17. Varni JW, Shulman RJ, Self MM, Nurko S, Saps M, Saeed SA, Bendo CB, et al. Symptom Profiles in Patients With Irritable Bowel Syndrome or Functional Abdominal Pain Compared With Healthy Controls. J Pediatr Gastroenterol Nutr. 2015;61(3):323-329.

18. Rasquin A, Di Lorenzo C, Forbes D, Guiraldes E, Hyams JS, Staiano A, Walker LS. Childhood functional gastrointestinal disorders: child/adolescent. Gastroenterology. 2006;130(5):1527-1537.

19. Chitkara DK, Rawat DJ, Talley NJ. The epidemiology of childhood recurrent abdominal pain in Western countries: a systematic review. Am J Gastroenterol. 2005;100(8):1868-1875.

20. Talachian E, Bidari A, Zahmatkesh H. Abdominal painrelated functional gastrointestinal disorders based on Rome III criteria in a pediatric gastroenterology clinic. Med J Islam Repub Iran. 2015;29:247.

21. Apley J. The child with recurrent abdominal pain. Pediatr Clin North Am. 1967;14(1):63-72.

22. Helgeland H, Flagstad G, Grotta J, Vandvik PO, Kristensen H, Markestad T. Diagnosing pediatric functional abdominal pain in children (4-15 years old) according to the Rome III Criteria: results from a Norwegian prospective study. J Pediatr Gastroenterol Nutr. 2009;49(3):309-315.

23. Rutten JM, Korterink JJ, Venmans LM, Benninga MA, Tabbers MM. Nonpharmacologic treatment of functional abdominal pain disorders: a systematic review. Pediatrics. 2015;135(3):522-535.

24. Sadeghian M, Farahmand F, Fallahi GH, Abbasi A. Cyproheptadine for the treatment of functional abdominal pain in childhood: a double-blinded randomized placebo- controlled trial. Minerva Pediatr. 2008;60(6):1367-1374.

25. Madani S, Cortes O, Thomas R. Cyproheptadine Use in Children With Functional Gastrointestinal Disorders. J Pediatr Gastroenterol Nutr. 2016;62(3):409-413.

26. Korterink JJ, Rutten JM, Venmans L, Benninga MA, Tabbers MM. Pharmacologic treatment in pediatric functional abdominal pain disorders: a systematic review. J Pediatr. 2015;166(2):424-431 e426.

27. Saps M, Youssef N, Miranda A, Nurko S, Hyman P, Cocjin J, Di Lorenzo C. Multicenter, randomized, placebo-controlled trial of amitriptyline in children with functional gastrointestinal disorders. Gastroenterology. 2009; 137(4):1261-1269.

28. Teitelbaum JE, Arora R. Long-term efficacy of low-dose tricyclic antidepressants for children with functional gastrointestinal disorders. J Pediatr Gastroenterol Nutr. 2011;53(3):260-264.

29. Phillips SF. Overview: the doctor-patient relationship. In: Camilleri M, Spiller RC, eds. Irritable Bowel Syndrome: Diagnosis and Treatment. Edinburgh, UK: Elsevier. 2002; p. 127-132.

30. Devanarayana NM, Mettananda S, Liyanarachchi C, Nanayakkara N, Mendis N, Perera N, Rajindrajith S. Abdominal pain-predominant functional gastrointestinal diseases in children and adolescents: prevalence, symptomatology, and association with emotional stress. J Pediatr Gastroenterol Nutr. 2011;53(6):659-665.

31. Locke GR, 3rd, Zinsmeister AR, Fett SL, Melton LJ, 3rd, Talley NJ. Overlap of gastrointestinal symptom complexes in a US community. Neurogastroenterol Motil. 2005;17(1):29-34.

32. Agreus L, Svardsudd K, Nyren O, Tibblin G. Irritable bowel syndrome and dyspepsia in the general population: overlap and lack of stability over time. Gastroenterology. 1995;109(3):671-680.

33. Halder SL, Locke GR, 3rd, Schleck CD, Zinsmeister AR, Melton LJ, 3rd, Talley NJ. Natural history of functional gastrointestinal disorders: a 12-year longitudinal population-based study. Gastroenterology. 2007;133(3):799807.

34. Gieteling MJ, Bierma-Zeinstra SM, Passchier J, Berger MY. Prognosis of chronic or recurrent abdominal pain in children. J Pediatr Gastroenterol Nutr. 2008;47(3):316326.

35. Wu JC. Psychological Co-morbidity in Functional Gastrointestinal Disorders: Epidemiology, Mechanisms and Management. J Neurogastroenterol Motil. 2012;18(1):1318.

36. Grimes DA, Schulz KF. Descriptive studies: what they can and cannot do. Lancet. 2002;359(9301):145-149. 